\title{
Agente de monitoramento de infraestrutura de redes para suporte a sistemas self-healing
}

\author{
Henrique P. T. Rabelo ${ }^{1}$, Felipe C. Costa Alves ${ }^{1}$, Jean Caminha ${ }^{1}$, Renan Susuki ${ }^{1}$, \\ Allan Gonçalves ${ }^{1}$, Hernane Junior ${ }^{1}$, Tierry Lincoln ${ }^{1}$ \\ ${ }^{1}$ Ponto de Presença da RNP em Mato Grosso - (POP-MT) \\ CEP 78060-900 - Cuiabá - MT - Brazil \\ \{henrique, felipe, jean, renan, allan, hernane, tierry\}@pop-mt.rnp.br
}

\begin{abstract}
Systems supported by computer network infrastructures are at risk of downtime caused by power outages and communications, causing business impacts. In order to detect and act upon degradation events of these services, we present an intelligent IoT-based monitoring agent.
\end{abstract}

Resumo. Os sistemas suportados pelas infraestruturas de redes de computadores sofrem com o risco de indisponibilidade ocasionados por quedas nos serviços de fornecimento de energia elétrica e comunicaçoes, causando impactos aos negócios. Com a finalidade de detectar e atuar em eventos de degradação destes seviços, apresentamos um agente de monitoranto inteligente baseado em Internet das Coisas.

\section{Introdução}

A indisponibilidade dos serviços de fornecimento de energia e comunicaçao é ainda um problema recorrente no Brasil, especialmente em regiões como o Centro-Oeste. Dados da Agência Nacional de Energia Elétrica (ANEEL) ${ }^{1}$ mostram que essas quedas podem chegar até a dez vezes em uma mesma unidade consumidora no Estado de Mato Grosso no período de um ano. Quedas frequentes de energia impactam datacenters e sistemas críticos de informações [Marin 2016].

As fontes de alimentação ininterrupta (UPS) são as soluções mais utilizadas para o tratamento de falhas no fornecimento de energia, entretanto esses sistemas não são projetados para identificar o local dos eventos de indisponiblidade. Interrupções no fornecedor do serviço (datacenters), redes de transporte ou até mesmo no usuário impactam diretamente o uso do serviço.

Uma alternativa para contornar essa limitação é o uso de sistemas baseados na filosofia self-healing, capazes de se auto diagnosticarem e reagirem de modo satisfatório para se restaurarem automaticamente após uma falha ou defeito[Shirazi and Jadid 2019].

O uso de internet das coisas, que é uma detecção onipresente de vários aspectos do ambiente por meio de uma rede de sensores sem fio com a capacidade de medir, inferir e entender os indicadores propostos[Gubbi et al. 2013], pode ser utilizada para auxiliar o monitoramento e executar as ações propostas pelo self-healing.

\footnotetext{
${ }^{1}$ http: //www2 .aneel.gov.br/aplicacoes/indicadores_de_qualidade/ decFecSegAnual . cfm? ano=2018\&REGIAO=CO\&DISTRIBUIDORA=4 05 \& ipo=d
} 
Com o objetivo de automatizar a detecção dos eventos e atuar no retorno das comunicações o mais rápido possível, apresentamos um agente de monitoramento inteligente de infraestrutura de redes baseado em self-healing e internet das coisas.

\section{Agente de monitoramento de infraestrutura de redes para suporte a sistemas self-healing}

O agente de monitoramento de infraestrutura de redes baseado em IoT para Self-healing 1(b) é um sistema composto por componentes responsáveis por monitorar a infraestrutura de rede do cliente, coletando, tratando e armazenando informações como quedas de energia, qualidade dos links, temperatura e umidade do ambiente (Figura 1 (a)).

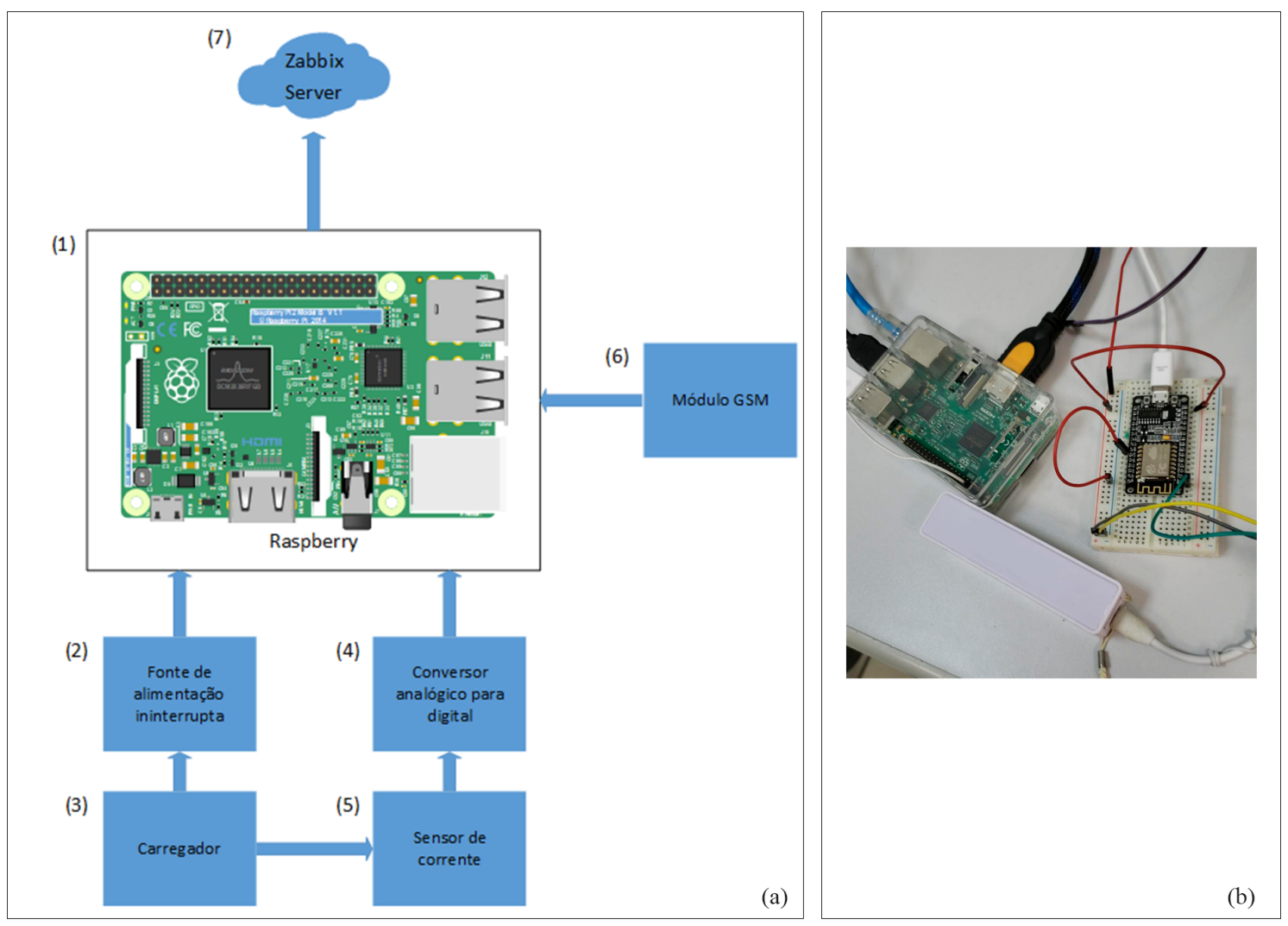

Figura 1. Arquitetura e protótipo do agente

O principal componente da arquitetura do agente é o Raspberry Pi (1) que é responsável por receber e tratar os dados, com auxílio do conversor analógico para digital (4) pois o Raspberry não consegue tratar dados analógicos. O principal sensor do sistema é o sensor de corrente (5) que monitora eventuais quedas de energia medindo a corrente que passa através do carregador (3), este por sua vez é responsável por manter a bateria da fonte de alimentação ininterrupta (2) sempre carregada. Junto dos sensores fez-se necessário a utilização de um módulo GSM (6) que fornece para o agente conexão móvel para utilizar de internet $3 \mathrm{G}$ caso ocorra uma eventual queda de energia. Uma vez que os dados são lidos e tratados devidamente são enviados para um servidor do software Zabbix (7), onde estas informações serão guardadas e analisadas com intuito de prever e evitar incidentes futuros. 


\section{Implementação}

A implementação do agente utiliza protocolos de comunicação e monitoramento abertos e de uso comum na internet, como o SNMP (Simple Network Management Protocol) responsável pelo gerenciamento da rede, tendo como intermediário a ferramenta NodeRED responsável por gerenciar as comunicações entre os componentes e como elas se comportam, com o uso das linguagens de programação Python, JavaScript e C.

\section{Resultados preliminares}

Os resultados em laboratório mostram que o protótipo do agente foi capaz de detectar quedas de energia imediatamente após o acontecimento e, com o uso do NodeRED, enviar notificações por e-mail com hora e data do ocorrido, além de enviar informações para o servidor do Zabbix, que é uma ferramenta open-source que utiliza, dentre diversos protocolos de monitoramento e comunicação, o SNMP para monitoramento de rede, por meio de arquivos com o formato JSON. Uma vez que estas informações passam pelo NodeRED, é póssivel executar as ações de recuperação devido a grande quantidade de nodes que o mesmo disponibiliza, facilitando a implementação das soluções[Seeger et al. 2019].

\section{Trabalhos futuros}

Os próximos passos no desenvolvimento do agente são: A implementação do monitoramento da qualidade dos links; a gama de sensores para coleta de informações do ambiente como temperatura e umidade; detecção dos problemas existentes na rede e propostas de soluções por meio do self-healing; além da configuração de uma segunda UPS para reserva caso a primeira falhe.

\section{Referências}

Gubbi, J., Buyya, R., Marusic, S., and Palaniswami, M. (2013). Internet of things (iot): A vision, architectural elements, and future directions. Future Generation Computer Systems, 29(7):1645 - 1660. Including Special sections: Cyber-enabled Distributed Computing for Ubiquitous Cloud and Network Services Cloud Computing and Scientific Applications — Big Data, Scalable Analytics, and Beyond.

Marin, P. S. (2016). Data Centers Engenharia: Infraestrutura Física. PM Books, 1 edition.

Seeger, J., Bröring, A., and Carle, G. (2019). Optimally self-healing iot choreographies. CoRR, abs/1907.04611.

Shirazi, E. and Jadid, S. (2019). A multiagent design for self-healing in electric power distribution systems. Electric Power Systems Research, 171:230 - 239. 